
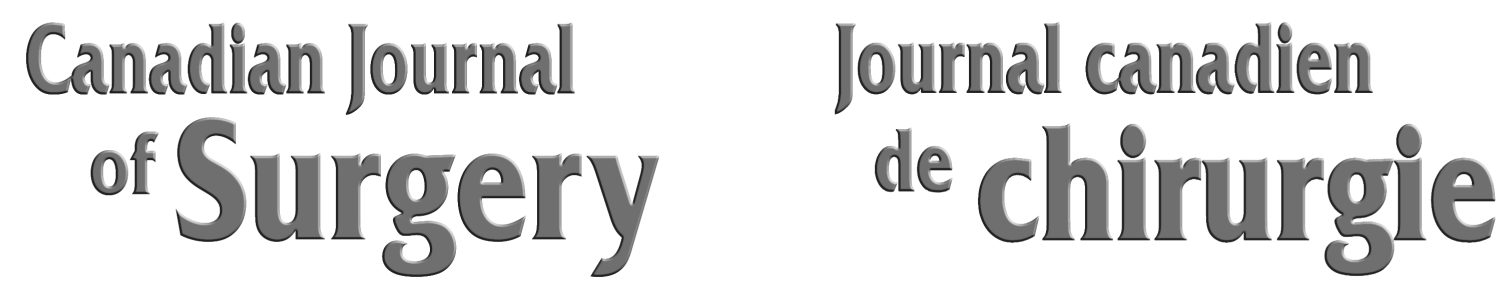

Vol. 56 (6 Suppl 1) December/décembre 2013 cma.ca/cjs DOI: $10.1503 /$ cjs.021513

\title{
Canadian Society for Vascular Surgery Annual Meeting on Vascular Surgery
}

\section{The Westin Edmonton Hotel Edmonton, Alberta}

Friday, Sept. 13 to Saturday, Sept. 14, 2013 


\section{Canadian Society for Vascular Surgery abstracts}

\author{
FridAY, SEPT. 13, 2013 \\ PAPer Session I: Aortic intervention
}

1

The predictive role of negative initial imaging for the development of an endoleak after infrarenal endovascular aortic aneurysm repair. S. Ladowski, H.L. Gill, K.S. Mackenzie, M.M. Corriveau, C.Z. Abraham, D.I. Obrand, O.K. Steinmetz. From the Division of Vascular Surgery, McGill University, Montréal, Que.

Background: The risk of endoleak after infrarenal endovascular aortic aneurysm repair is significant and thus patients require lifelong imaging surveillance. This surveillance comes with its own set of risks associated with radiation exposure and contrast dye use, as well as increased costs. We sought to determine the predictive value of a negative first postoperative imaging study on the long-term risk of developing an endoleak in a varied tertiary care vascular practice. We sought to determine if there are characteristics that might lend a subgroup of patients to be able to follow less rigorous imaging protocols. Methods: We completed a retrospective review of prospectively collected institutional outcomes data (2004-2009), including stratification according to postoperative imaging results, placing them into 2 groups: 1) normal first postoperative computed tomography angiography (CTA) and 2) endoleak on first postoperative CTA. Baseline characteristics and aneurysm morphology were compared between the 2 groups. Multivariate models were used to identify risk factors for development of endoleak and to assess the predictive value of a negative first postoperative imaging test. Results: A total of 134 patients were included in the analysis. Patients were treated with a mixture of Aorfix $(n=2)$, Endurant $(n=23)$, Talent $(n=86)$, and Zenith $(n=23)$ stent grafts. The median follow-up time was 2.51 years (interquartile range 1.2$4.09 \mathrm{yr}$ ). During this period, a total of 33 patients were found to have an endoleak and 12 patients underwent secondary interventions for those leaks. On first postoperative CTA (mean $28 \mathrm{~d}$ after intervention), 27 patients (20\%) had an endoleak while 107 did not. Those patients with a normal first postoperative scan were significantly less likely to go on to develop an endoleak (5.6\% v. $22.2 \%, p=0.007)$, to require a secondary intervention for treatment of an endoleak (4.7\% v. $25.9 \%, p=0.001)$ and to go on to develop aneurysm expansion $(7.5 \%$ v. $22.2 \%, p=0.025)$. Conclusion: These data suggest that some patients whose first postoperative CT is normal post-EVAR may be candidates for less rigorous postoperative screening protocols. Further evaluation of patient and aneurysm characteristics would be needed before safely applying such a protocol in clinical practice.

\section{2}

Transesophageal echocardiogram and use of contrast during proximal aortic endograft placement: a comparison of contrast TEE with completion aortogram and postoperative CT angiography. M.E. MacDonald, ${ }^{*}$ R.D. Moore, ${ }^{*}$ G.M. Dobson. ${ }^{\dagger}$ From the *Division of Vascular Surgery and the tDepartment of Anaesthesia, Alberta Health Services, Calgary, Alta.
Background: Thoracic endovascular aortic repair (TEVAR) has become a widespread method of aortic repair with distinct advantages over open repair in the elective and emergency setting. The primary technical consideration is achievement of endoseal, which is limited by aortic arch curvature and branch vessel anatomy. Our objective was to evaluate whether the use of transesophageal echocardiogram (TEE) with ultrasound contrast as an intraoperative adjunct to arteriography may achieve greater overall sensitivity for endoleak while limiting the use of intravenous contrast. Methods: In this retrospective review, we compared TEE with colour-flow Doppler and TEE with contrast to the current imaging standards, completion aortography and postoperative CT angiogram. Results: There were 39 TEVAR cases in which TEE with contrast was used for intraoperative assessment. Two of these were emergent (rupture) TEVARs. Other indications included type B aortic dissection after failure of medical management ( 5 cases), stage 2 TEVAR for type A dissection (1), thoracic aortic aneurysm (20), penetrating aortic ulcer (7), symptomatic aortic pseudoaneurysm (3), and Kommerell diverticulum in a right-sided aortic arch (1). Transesophageal echocardiography was used to rule out endoleak in all cases and contributed as well to the following 51 intraoperative decisions: guiding wire into the true lumen of a type $\mathrm{B}$ dissection (5), guiding proximal deployment of a stent (15), identifying endoleak, guiding balloon deployment and confirming resolution of endoleak (16, including 4 leaks not seen on intraoperative angiography), confirming branch patency (11), placing another stent (2), and opting for no further interventions (2). There were no instances of endoleak seen only on intraoperative angiography (i.e., no endoleaks missed by TEE with ultrasound contrast) in this series. Conclusion: Review of all TEVAR cases done by the Division of Vascular Surgery in Calgary, Alta., from 2004 to 2012 demonstrates that TEE with contrast aids in intraoperative decision-making, including detection of endoleak and branch patency.

\section{3}

Surgeon efficiency is the most important predictor of fluoroscopy duration: Anatomic and procedural determinates of fluoroscopy time during elective EVAR. R. Ruz, L. Dubois, K. Lee, A.H. Power, J.R. Harris, G. DeRose, T.L. Forbes. From the Division of Vascular Surgery, Western University, London, Ont.

Background: Our objective was to identify both the procedural and anatomic factors that determine duration of fluoroscopy during EVAR. Methods: We retrospectively analyzed our prospectively maintained EVAR database for the relationship between fluoroscopy time and both procedural (i.e., type of graft, configuration, number of components, surgeon) and anatomic factors reflective of aneurysm complexity (i.e., 17 variables derived from the validated St. George's protocol). Linear regression was used to identify significant predictors of fluoroscopy time. Results: A total of 128 patients underwent elective EVAR between October 2011 and February 2012 with a mean fluoroscopy time of $5.7 \pm 3.4$ minutes. The grafts used comprised 41 (32\%) Zenith (Cook) and 85 (66\%) Endurant (Medtronic), with 105 (82\%) bifurcated and 23 (18\%) aorto-uni-iliac (AUI) in configuration. The mean number of components used was 2.6, with 
4 surgeons performing the procedures. Both the surgeon performing the procedure $(p=0.001)$ and graft configuration (bifurcated v. AUI, $p=0.03$ ) were found to be predictive of fluoroscopy time, while number of components inserted, type of graft, and anatomic variables (e.g., lengths, diameters, tortuosity, calcification) had no effect on fluoroscopy duration. Mean surgeon fluoroscopy time ranged from $4.5 \pm 1.8$ minutes to $7.5 \pm 5.1$ minutes, while mean fluoroscopy time was lower for aorto-uni-iliac versus bifurcated devices $(4.5 \pm 2.6 \mathrm{~min}$ v. $5.9 \pm 3.5 \mathrm{~min})$. Conclusion: The surgeon's efficiency in the use of fluoroscopy during EVAR is the most important determinant of total fluoroscopy time. Anatomic complexity, make of device, and number of components inserted have minimal impact on duration of fluoroscopy. An endovascular surgeon's ability to curtail fluoroscopy duration is the key component in minimizing radiation exposure to both the surgical team and the patient. Given its strong association with the individual surgeon, fluoroscopy time may evolve as a quality measure for those performing EVAR.

\section{4}

Iliac branch device procedures: Does anatomy matter? D. Jiang, J. Gagnon, J. Chen. From the Division of Vascular Surgery, University of British Columbia, Vancouver, BC

Background: Our objective was to review the iliac branch device (IBD) procedures performed at Vancouver General Hospital and to evaluate their outcomes. Methods: A retrospective review was conducted of patients who underwent endovascular aneurysm repairs incorporating IBDs from May 2008 to February 2012. Data on patient demographics, comorbidities, anatomic details, postoperative events and follow-up findings were gathered by reviewing hospital charts, clinic charts as well as perioperative and postoperative imaging. Results: A total of 23 procedures were reviewed. One patient received bilateral IBD procedures, which were counted separately. Follow-up time ranged from 1 month to 39 months (median 12). The average patient age was 78.9 years. Twelve procedures were done in accordance with instructions for use (IFU). Six procedures had extension of the branch graft into the posterior branch of the internal iliac artery (POST); 5 procedures had smaller than recommended $(<16 \mathrm{~mm})$ distal common iliac artery diameter (SmCI). The overall technical success rate was $95.7 \%$. One patient in the SmCI group had intraoperative failure of the IBD requiring an open external iliac artery to internal iliac artery (IIA) bypass. Overall branch patency rates at 1 year and 2 years were $85.9 \%$ and $73.7 \%$, respectively. Upon follow-up, there was 1 IIA occlusion in the IFU group (8.3\%), 3 in the POST group $(50 \%)$ and 1 in the SmCI group (20\%). There were 5 flowlimiting external iliac artery stenoses requiring endovascular intervention (21.7\%). There were 2 type IB endoleaks present on follow-up computed tomography angiography. One type III endoleak proximal to the IBD caused aortic aneurysm rupture requiring urgent endovascular repair. Our overall reintervention rate was $34.8 \%$ ( 8 of 23 ). Fourteen percent (3 of 22) of patients had ipsilateral claudication at the last follow-up. Conclusion: Our IBD experience yielded suboptimal results with a higher than expected reintervention rate. Nonadherence to IFU appears to have a negative impact.

\section{5}

Predictors of abdominal aortic aneurysm sac enlargement after endovascular repair in the greater Toronto area. L. Figueroa-Gallaga, ${ }^{*}$ M. Pope, ${ }^{+}$A. Moloney, G. Roche-Nagle. ${ }^{*}$ From the *Division of Vascular Surgery,
Toronto General Hospital, University of Toronto, †Trillium Health Science Centre, and the $¥$ Division of Vascular Surgery, St. Michael's Hospital, University of Toronto, Toronto, Ont.

Background: Our objective was to evaluate compliance with instructions for use and sac enlargement rate after endovascular abdominal aortic aneurysm repair. Methods: All patients that underwent endovascular aneurysm repairs with a preoperative computerized tomographic angiography (CTA) as well as a postop follow-up with either CTA or ultrasound (US) will be enrolled in this observational study. Demographic data will be obtained. Preoperative aortoiliac characteristics are assessed using Vitrea FX abdominal aortic aneurysm (AAA) worksheet (Toshiba Vital, version 6.3) and the latest follow up measurements as reported in the CTA or US. Secondary interventions are also assessed and reported. Results: The data presented in this abstract is partial, including patients from one of the sites, Toronto General Hospital (2010-2011); further data collection is commencing at the other sites. A total of 143 patients have been analyzed, of which 118 (82\%) were male. Elective aneurysm repair was performed in $106(74 \%)$ cases, urgent in 37 patients (26\%), of which $15(10 \%)$ were symptomatic abdominal aortic aneurysm (AAA) and $22(15 \%)$ were ruptured AAA. Cook devices were used in 114 cases (80\%), Medtronic in 25 (17\%) and Anaconda in $4(3 \%)$. The mean maximum sac diameter was $59 \mathrm{~mm}$ (range 50-90.5) and mean sac volume was 153 cc (range 60.5347). The mean aortic neck length was $25 \mathrm{~mm}$ (range 5.5-58); mean diameter measured $23 \mathrm{~mm}$ (range 19-33.5) and mean angulation measured $35^{\circ}$ (range 9-65.2). After endovascular aneurysm repairs, the mean sac enlargement was $-5.7 \mathrm{~mm}$ (range -14.3 to 6.7) with AAA maximum sac diameter of $51.3 \mathrm{~mm}$ (range $36-72$ ). The endoleak rate was $4.4 \%$ with 5 type 1, 26 type II and 1 type $\mathrm{V}$, with no type III or IV endoleaks. After secondary interventions all type I endoleaks were successfully treated. Conclusion: To date we have demonstrated better compliance with published IFUs in comparison to previously published data. Further analysis will determine if this is associated with better outcomes.

\section{6}

EVAR stent migration and late onset type II endoleaks. I. Syed, A.M. Berner, M. Jumah, P. Bandorawalla. From the Interventional Radiology Department, Barking, Havering \& Redbridge University Hospitals NHS Trust, UK

Background: Stent migration following EVAR is related to complications including endoleak. The CSVS standards define migration as over $10 \mathrm{~mm}$, but data regarding incidence of migration and its relationship to endoleak is sparse. The aim of this study was to investigate migration of EVAR stents postprocedure and correlation with endoleaks. Methods: The CT angiographies of patients who underwent an EVAR at our centre between March 2006 and September 2011 were retrospectively studied. All included patients had CT angiograms less than 1 year post-EVAR and interval follow-up CT over 300 days. Maximum anteroposterior (AP) diameter of aneurysm was measured on pre-EVAR CT scan. Post-EVAR and most recent CT scans were examined for proximal stent migration (defined as a change in distance from 2 fixed points: superior mesenteric artery to top of bare metal stent) and for evidence of endoleak(s). Results: A total of 76 patients were studied. Mean proximal caudal migration was $1.55 \mathrm{~mm}$ (95\% CI $0.70-2.40)$. Ten patients had evidence of type II endoleaks on most recent CT. There was an increased risk of type II endoleaks on most-recent CT scan with 
caudal migration of the stent (OR 1.60, 95\% CI 8.35-0.31). Average AP diameter of aneurysm was slightly higher for patients with an endoleak on most recent CT scan compared with those without $(62.85 \mathrm{~mm}, 95 \%$ CI 62.71-63.99 v. $59.00 \mathrm{~mm}, 95 \%$ CI 58.91-59.09). Conclusion: Our study demonstrated minimal stent migration and overall late onset endoleaks are a rare event. Although there is a positive correlation between stent migration and type II endoleaks, a larger sample population is required to validate this finding due to low incidence of endoleaks detected. No type I endoleaks were found. Patients with endoleaks have a significantly higher mean AP diameter of aneurysm, but again larger studies would be required to further investigate this correlation.

\section{FridAY, SEPT. 13, 2013}

\section{PAPER SESSION II: Abdominal AORTIC ANEURYsm}

\section{7}

Retroperitoneal abdominal aortic aneurysm repair. A single centre, single surgeon's experience in 251 consecutive cases. G. Hajjar, T. Brandys, A. Hill, P. Jetty, S. Nagpal. From the Division of Vascular and Endovascular Surgery, the Ottawa Hospital, Civic Campus and the University of Ottawa, Ottawa, Ont.

Background: Both anecdotal and objective reports exist, as to the advantages of retroperitoneal approach to repair of abdominal aortic aneurysms (AAAs). It is considered the approach of choice for difficult aneurysm necks or hostile abdomens. In this series we reviewed the outcome of this approach in 251 consecutive patients at a single centre, with a variety of complicated anatomic features. Methods: Retrospective review of 251 consecutive patients between 2004 and 2012. All abdominal aortic aneurysms undergoing open repair were included. Unstable acute ruptures with large hematoma in left lower quadrant, and patients needing concomitant right renal or mesenteric bypass were excluded. Results: A total of 251 patients were reviewed, of which 195 were male and 56 were female. The mean age was 71 years. Comorbidities included coronary artery disease (112), chronic obstructive pulmonary disease (40), diabetes (44), obesity $(\mathrm{BMI}>30 ; 46)$, smoking (230) and hypertension (165). Aneurysm characteristics included primary (246), redo (5), electives (231), acute/symptomatic (15) and ruptured (5). Aneurysm morphology included infra-renal (212), Juxta-renal (33), suprarenal (6). Eighty-nine patients had associated iliac aneurysms, 24 had aortoiliac disease requiring an access blood flow graft, 6 were inflammatory type and 2 had horseshoe kidneys. Additional procedures included renal reimplantation or graft. Two patients had preliminary ax-fem, excision of infected aortic graft and duodenal closure, while 5 had inferior mesenteric artery reimplantation. Intraoperative mortality was 0 , while 30 -day mortality was 2 and total in-hospital mortality was $4(1.6 \%)$. The average length of stay in-hospital was 6 days. Complications included cardiac arrhythmias (12), congestive heart failure (10), myocardial infarction (8), transient rise in creatinine (20), acute renal failure ( 2 hemofiltration only, 3 transient dialysis, 1 permanent), respiratory exacerbation of chronic obstructive pulmonary disease (5) and pulmonary embolism (1). Postoperative bleeding occurred in 6 patients. Other complications included ischemic colitis (8 transient, 4 C. difficile, 2 colectomies), upper gastro intestinal bleeding (6 gastroduodenal erosions, 4 postop ileus), peripheral ischemia (4), incisional complications (13), wound infection (6), wound hematoma (4), serosanguinous drainage (3), and late incisional complications ( 1 intercostal neuralgia, 6 spe- cific complaints about asymmetric bulge). Conclusion: In this series, retroperitoneal approach to repair of abdominal aortic aneurysm compares favourably to published data on open transabdominal repair. It is easily feasible for a variety of complicated anatomic situations, and should not be restricted to just difficult upper ends or hostile abdomens.

8

Vascular and nonvascular reinterventions after open abdominal aortic aneurysm repair. B. Cartier. From CSSS du Suroît, Valleyfield, Que.

Background: The perioperative mortality of abdominal aortic aneurysm (AAA) repair is low with the endovascular and open approach, although with a slight advantage for EVAR; however, the rate of reintervention for endovascular aneurysm repair can reach $20 \%$ with $2 \%$ mortality. But what is the rate of reintervention in open surgery? The author relates his personal experience. Methods: This is a retrospective study of abdominal aortic surgery performed by the author between 2000 and 2011. During this period, 302 aortic surgeries were performed including 238 for AAA. Of these AAA surgeries, 29 were for ruptured AAA and 11 for iliac aneurysms. Eighteen patients were excluded because the follow-up was less than 1 month. Results: Of 220 patients, 46 were women (55-87 yr) and 174 were men (47$88 \mathrm{yr}$ ). The dimension of the AAA ranged from $4.6-12 \mathrm{~cm}$. Follow-up time ranged from 1-167 months (average 30.3). Comorbidities were $78.2 \%$ with previous abdominal surgery, including mast cell activation syndrome (23.2\%), hypertension (66\%), Db (16.4\%), hyperlipemia (53.4\%), chronic obstructive pulmonary disease $(14.3 \%)$, smoking $(47 \%)$, obesity $(1.3 \%)$. Perioperative morbidity for over 238 patients were cardiac (29.4\%), pulmonary $(8 \%)$ and renal $(7.1 \%)$ morbidities. Perioperative mortality was 7 of 209 for elective surgery $(3.3 \%)$ and 9 of 29 for emergency surgery (31\%). Incisional hernia at followup occurred for 39 of 220 patients $(17.7 \%)$; of these patients, 18 required treatment for hernia $(8.2 \%$, of 220$)$. Nine of the 220 patients were admitted for subocclusion (4\%), including 3 requiring surgery and 1 resulting in death. There were 12 vascular reinterventions (9 graft-related) in 9 patients, and 1 death. In total, there were 28 reinterventions (9 vascular, 19 nonvascular) in 26 patients $(11.8 \%)$. Of the 220 total, there were 2 deaths $(0.9 \%)$. Conclusion: This study showed a low rate of nonvascular and vascular reinterventions after open AAA repair and low long-term mortality related to the procedure.

\section{9}

An analysis of extended length of stay of patients undergoing elective infrarenal EVAR: a local study. N. Eisenberg, G. Roche-Nagle, G. Oreopoulos. From the Division of Vascular Surgery, University Health Network, Toronto, Ont.

Background: The Division of Vascular Surgery at the University Health Network in Toronto (UHN) has been a member of the Canadian Society for Vascular Surgery's Vascular Quality Initiative (SVS-VQI) since August 2010. It was identified by SVSVQI that our length of stay (LOS) is longer than expected for elective infrarenal endovascular aneurysm repair (EVAR) when compared with VQI benchmarking data from other centres $(\leq 2 \mathrm{~d})$. Extended LOS is associated with an increased risk of hospital-acquired morbidity and increased costs. The purpose of this retrospective study was to identify factors that contribute to 
extended LOS for patients undergoing standard, infrarenal EVAR procedures at UHN, and establish a quality improvement framework for addressing those findings. Methods: Institutional Research Ethics Board approval was obtained. We identified EVAR cases entered between January 2011 and December 2012 and performed a detailed chart audit to capture factors that may have contributed to an extended LOS. Physician office charts and hospital electronic records were used. We included and compared patients who stayed for the expected duration and those who stayed beyond. Emergency cases, complex and thoracic EVARs were excluded. We evaluated the univariate associations between all categorical variables and longer LOS (>2 d) using the $\chi^{2}$ or Fisher exact test. Results: One hundred and forty-four charts were identified for the time period, and 31 (21\%) were excluded (ruptured or symptomatic), leaving 113 charts for analysis. We found that 59 patients (52\%) stayed longer than 2 days. Statistically significant factors identified as being important were abnormal vital signs POD 0 , cardiovascular, wound and urologic issues. There was a trend toward advanced age (> $80 \mathrm{yr}$ ), making it a significant factor. Conclusion: Multiple factors were identified as contributing to extended LOS. Efforts to mitigate the effects of these factors in the perioperative setting may decrease our LOS and complication rate. Use of a centralized, benchmarking database has been informative in guiding our quality improvement efforts.

\section{0}

Institutional experience with short-stay EVAR. J. McDonald, ${ }^{*}$ M. Guirgis, ${ }^{*}$ F. Naji, ${ }^{\dagger}$ D.A. Szalay. ${ }^{*}$ From the *Division of Vascular Surgery, McMaster University, and the +Michael G. DeGroote School of Medicine, McMaster University, Hamilton, Ont.

Background: Endovascular aortic aneurysm repair is a wellestablished treatment modality in the management of infrarenal abdominal aortic aneurysms (AAA), offering lower morbidity and mortality as well as reduced overall hospital length of stay (LOS). Many of our postop EVAR patients have an LOS of only 1 day, and a smaller subset of that group is planned shortstay/overnight (SSO) admissions. With an increasing focus on resource management, SSO EVARs can potentially reduce overall hospital costs as well as permit a greater number of patients treated. The aim of this study was to assess the safety and efficacy SSO EVARs compared with conventional non-SSO EVARs. Methods: Retrospective review of patients who underwent EVAR for infrarenal AAA between January 2012 and February 2013 took place at our institution. Exclusion criteria included fenestrated, unilateral or iliac-branched grafts, as well as EVARs for ruptured AAA. Demographics, comorbidities, LOS, 30-day readmission rates, complications and mortality data were recorded. Descriptive and logistic regression was performed. Results: Between January 2012 and February 2013, 144 EVARs met our inclusion criteria. Overall average LOS was 1.93 days with a 30 -day readmit rate of $3.47 \%(n=5)$. There were 127 non-SSO EVARs with an average LOS of 2.05 days and a 30-day readmit rate of $3.14 \%(n=4)$. Of these 127 non-SSO EVARs, $65 \%(n=83)$ had an LOS of only 1 day and a 30 -day readmit rate of $2.41 \%(n=2)$. Alternatively, there were 17 SSO EVARs (4 of which failed and required a longer hospital stay), with an average LOS of 1.06 days and a 30-day readmit rate of $5.88 \%(n=1)$. Overall there were 96 EVARs with an LOS of 1 day (83 non$\mathrm{SSO}+13$ successful SSO), in which the 30 -day readmit rate was $3.13 \%(n=3)$. Conclusion: In select patients, 1 -day hospital admission following EVAR can offer a safe, effective, and reduced LOS alternative to conventional EVAR hospital stays with a similar 30-day readmit rate.

\author{
FridAY, SEPT. 13, 2013 \\ PAPER SESSION III: VASCULAR EDUCATION AND BASIC \\ RESEARCH
}

\section{1}

Assessing uncertainty in vascular trainees in a seminar setting: use of a script concordance model. E.M. Wooster, ${ }^{*}$ D.L. Wooster. ${ }^{\dagger}$ From the ${ }^{*}$ Ontario Institute for Studies in Education (OISE), University of Toronto, and the tDivision of Vascular Surgery, Department of Surgery, University of Toronto, Toronto, Ont.

Background: Structured seminars are a common learning setting for vascular trainees. They are usually directed by faculty, anchored in case discussion and explore trainees' knowledge of the "right" answers. Such a learning model relies on activation of prior knowledge to synthesize an approach to a real clinical situation. Many practice situations are not well-defined and introduce an element of uncertainty. The script concordance model (SCM) allows for learning in context with development of rich knowledge structures. The aims of this study were to 1) address the potential use of SCM in a seminar setting, 2) determine if trainees could engage with the uncertainty presented and 3) identify if such engagement resulted in deeper understanding of the material. Methods: Trainees were presented with similar clinical topics in the setting of a traditional seminar with structured discussion and a seminar presented in an 'uncertainty' format with SCM questions. The trainees' attitudes to each format were noted by objective (i.e., discussion time, depth of enquiry and additional questions) and subjective (i.e., interest, attention, intent to learn) metrics. Results: Trainees attended 2 seminars in each format. Discussion time (22 v. $15 \mathrm{~min})$, depth of enquiry (3+ v. $1+$ ) and additional questions (12 v. 4) showed greater engagement with the SCM approach. Subjective measures favoured the SCM also. Conclusion: The SCM approach to explore uncertainty in the seminar setting showed positive outcomes in a variety of spheres. The study is limited by small numbers. Further study to identify learning benefits, retention of learning and application of learning in clinical situations is required.

\section{2}

Using an iPad multimedia presentation to improve patient understanding and satisfaction with informed consent for minimally invasive vascular procedures: a pilot study. N. Bowers, ${ }^{*}$ N. Eisenberg, ${ }^{+}$J. Jaskolka, ${ }^{\neq}$ G. Roche-Nagle. ${ }^{+}$From the ${ }^{*}$ Faculty of Medicine, University of Toronto, tDivision of Vascular Surgery, University Health Network, and the ¥Division of Vascular/ Interventional Radiology, University Health Network, Toronto, Ont.

Background: Vascular interventions are becoming increasingly complex. As procedures become more complex, patient understanding of their treatments can become more difficult. This study aims to evaluate the utility of multimedia presentations (MMP) on an iPad tablet computer for patient understanding of vascular interventions. Methods: Patients undergoing EVAR, peripheral angioplasty, Hickman catheter and peripherally inserted central catheter insertion were randomized into a control group receiving traditional verbal consent, and an MMP group that was shown a 2 -minute simplified video of the procedure on 
an iPad in addition to the traditional verbal consent. After obtaining consent, and before the procedure, all patients completed a questionnaire assessing their comprehension of the procedure and satisfaction with the consent process. Results: Ninety-three patients were recruited for this study. Patients in the MMP group reported the utility of the MMP as an average of 4.8 on a 5 -point Likert scale, with 5 being "very helpful" in understanding the procedure. The MMP was shown to improve patient comprehension of EVAR and angioplasty procedures, with EVAR patients in the MMP group answering more questions correctly $(p=0.04)$, and angioplasty patients in the MMP group answering more questions correctly $(p=0.002)$, than the verbal consent control groups during assessment of comprehension. Conclusion: This study has shown that patients find the use of MMP during the consent process to be very helpful in understanding vascular interventions, and that patients receiving certain vascular interventions have improved comprehension of their procedures when shown a MMP during the consent process. Given the rapid rate of innovation in vascular surgery, the use of MMP to help patients understand new and complex procedures would be beneficial in the future care of patients undergoing vascular interventions.

\section{3}

The effects of pulsatile fatigue on in situ antegrade fenestrated endovascular stent grafts deployed inside a semirealistic phantom of an abdominal aortic aneurysm. A. Ruthrauff, ${ }^{*}$ M.W. King, ${ }^{*}$ K.T. Tan, ${ }^{\dagger}$ G. Soulez, ${ }^{\ddagger}$ L.W. Tse. ${ }^{\S}$ From the *College of Textiles, North Carolina State University, Raleigh, N.C., †Division of Vascular/Interventional Radiology, Toronto General Hospital, Joint Department Of Medical Imaging, University Health Network, Toronto, Ont., ‡Département de radiologie, Hôpital Notre-Dame, CHUM, Université de Montréal, Montréal, Que., and the §Division of Vascular Surgery, Toronto General Hospital, Peter Munk Cardiac Centre, University Health Network, Toronto, Ont.

Background: Our objective was to observe the performance of conventional stent grafts after being fenestrated using radiofrequency (RF) puncture and a conventional angioplasty balloon or a cutting balloon, followed by pulsatile fatigue for 40 million cycles. Methods: Two Cook Zenith and 2 Medtronic Endurant devices were deployed suprarenally into polyurethane phantoms of an abdominal aortic aneurysm so that the renal arteries were covered. They were then fenestrated under fluoroscopy at the renal arteries using a radiofrequency puncture device and dilated using 2 different types of balloon catheters: a conventional angioplasty balloon for 1 of each type of device and a cutting balloon for the remaining 2 devices. Atrium stent grafts were deployed through the fenestrations. They were then fatigued on a pulsatile fatigue tester for 40 million cycles or the equivalent of 1 year in vivo. The devices were then examined for evidence of loss of integrity of the graft fabric and enlargement of the fenestrations. Results: A significant waist was observed on the Atrium extensions when a conventional angioplasty balloon was used. All devices showed signs of melted and fused fibres at the edge of the fenestration. After fatigue testing the extent of fraying was greater with use of a cutting balloon. None of the fenestration sizes or dimensions increased after fatiguing. As expected, the burst resistance of the fenestrated areas decreased, reflecting the relative size of the fenestration. After fatiguing, the radial stiffness increased for both Cook devices, and decreased for both Medtronic devices. Conclusion: There were changes in the structure due to both fenestration and fatigue but they did not impact the overall integrity of the devices. The type and extent of change were dependent on the material selection, fabric structure and the fenestration technique. There was no enlargement of the fenestrations after fatiguing.

14

Complement deficiency attenuates gastrointestinal ischemia reperfusion injury in a mouse model of ruptured abdominal aortic aneurysm. W.S. Johnson, T.F. Lindsay. From the Division of Vascular Surgery, University Health Network and Department of Surgery, University of Toronto, Toronto, Ont.

Background: Our objective was to examine the role of complement immune system activation in ischemia-reperfusion (I/R) injury after ruptured abdominal aortic aneurysm (RAAA) using a complement deficient knockout (K/O) mouse model. Methods: Wild type and complement deficient knockout mice were used to model the sequential I/R injuries of hemorrhagic shock and supramesenteric aortic clamping of RAAA repair. We employed complement deficient mice that were unable to activate the complement immune system by 1 of the 3 known initiation streams: mannan-binding lectin, classical, or alternative pathways. Gut and lung injuries were quantified as the degree of $\mathrm{I}^{125}$-labelled albumin leak. Neutrophil sequestration into the gut and lung were measured via levels of myeloperoxidase. Results: See Table. Conclusion: This model simulates the sequential I/R injuries associated with open repair of RAAA. It results in significant local reperfusion gut injury, perhaps mediated via neutrophil sequestration. Interference with complement immune system activation by targeted knockout of complement initiation significantly attenuates gastrointestinal I/R injury, and reduces neutrophil sequestration in both the gut and lung. Complement plays a significant role in the initiation of $I / R$ injury in this mouse model of RAAA, which suggests the complement pathway as a future target for investigation into the mechanisms of organ dysfunction after RAAA repair.

\begin{tabular}{|c|c|c|c|c|c|}
\hline \multirow[b]{2}{*}{ Factor } & \multicolumn{5}{|c|}{ Group; mean \pm SEM } \\
\hline & Sham & $\begin{array}{l}\text { Wild } \\
\text { type }\end{array}$ & $\begin{array}{c}\mathrm{MBL} \\
\mathrm{K} / \mathrm{O}\end{array}$ & $\begin{array}{l}\mathrm{C} 1 \mathrm{q} \\
\mathrm{K} / \mathrm{O}\end{array}$ & $\begin{array}{l}\text { Factor } \\
\text { B K/O }\end{array}$ \\
\hline $\begin{array}{l}\text { Gut permeability, } \\
\text { units/uL serum } 1^{125} \\
\text { activity/90 min of } \\
\text { reperfusion }\end{array}$ & $\begin{array}{c}1.64 \pm \\
0.53\end{array}$ & $\begin{array}{c}6.61 \pm \\
1.75^{*}\end{array}$ & $\begin{array}{c}3.32 \pm \\
0.69\end{array}$ & $\begin{array}{c}1.92 \pm \\
0.21\end{array}$ & $\begin{array}{c}2.84 \pm \\
0.35\end{array}$ \\
\hline $\begin{array}{l}\text { Gut neutrophil } \\
\text { sequestration, } \\
\text { MPO/mg gut } \\
\text { protein }\end{array}$ & $\begin{array}{c}0.20 \pm \\
0.05\end{array}$ & $\begin{array}{l}0.38 \pm \\
0.04^{*}\end{array}$ & $\begin{array}{c}0.27 \pm \\
0.06\end{array}$ & $\begin{array}{c}0.05 \pm \\
0.01\end{array}$ & $\begin{array}{c}0.07 \pm \\
0.01\end{array}$ \\
\hline $\begin{array}{l}\mathrm{C} 1 \mathrm{q}=\text { classical } ; \mathrm{K} / \mathrm{O}= \\
\text { peroxidase; } \mathrm{SEM}=\text { sta } \\
{ }^{*} p<0.05 \mathrm{v} \text {. Sham. }\end{array}$ & $\begin{array}{l}\text { tout; } \mathrm{ME} \\
\text { error of }\end{array}$ & $\begin{array}{l}=\text { mannar } \\
\text { mean. }\end{array}$ & & & \\
\hline
\end{tabular}

\section{5}

Elevated A1c is a predictor of poor outcome following vascular surgery. A.B. Hill, H. Khambati, P. Jetty, G. Hajjar, T. Brandys, S. Nagpal. From the Division of Vascular and Endovascular Surgery, the Ottawa Hospital, Civic Campus and the University of Ottawa, Ottawa, Ont. 
Background: Diabetes is present in $30 \%$ to $40 \%$ of patients presenting for vascular surgery. Diabetes and hyperglycemia have been implicated as risk factors for perioperative complications in other study populations; however, there is limited data concerning the role of glycemic control in the vascular population. Methods: A1c, a marker of glycemic control in diabetics, was measured before surgery in 520 consecutive patients undergoing vascular surgery between August 2009 and December 2010. The patients were evaluated for demographic data, glucose levels and perioperative complications. Results: Of the 169 patients with diabetes, $60 \%$ had elevated A1c (> 0.065) before surgery. Patients with A1c levels $>0.065$ had increased risk of stroke or a coronary event following surgery and the risk appears to be related to the degree of preoperative hyperglycemia (A1c > 0.065 , relative risk [RR] 2.4, $p=0.03$; A1c $>0.75$, RR 3.2, $p=$ 0.01; A1c $>0.08, \mathrm{RR} 4.2, p<0.01$ ). The effect of elevated A1c on stroke or coronary events was independent of other risk factors $(p=0.03$, logistic regression analysis). Complication free surgery was predicted by normal A1c levels while A1c $>0.08$ predicted morbidity and mortality following vascular surgery (RR 1.5, $p=$ 0.04). The development of complications was associated with increased length of hospital stay: mean 31.2 days (95\% CI 25.336.9 ) versus 7.3 days (95\% CI 6.5-8.2) for uncomplicated surgery patients. Conclusion: A significant proportion of diabetics present for vascular surgery with poorly controlled glucose. Elevated preoperative A1c and hyperglycemia predicts poor outcome for patients undergoing vascular surgery. Strategies to identify and manage hyperglycemia may provide an opportunity to improve patient outcome following vascular surgery.

\section{Saturday, SePt. 14, 2013 Paper Session IV: Peripheral Vascular disease}

\section{6}

Angioplasty or primary stenting for infrapopliteal arterial occlusive disease: a meta-analysis. M. Qadura, F. Elias, M. Guirgis, A. Saleh, T. Rapanos, D. Szalay, J. Harlock. From the Division of Vascular Surgery, McMaster University, Hamilton, Ont.

Background: There has been a significant development in the endovascular treatment of infrapopliteal peripheral arterial disease. This meta-analysis pooled all of the randomized clinical trials (RCTs) that compared percutaneous transluminal angioplasty (PTA) versus PTA plus stenting approaches for treatment of patients with infrapopliteal chronic critical limb ischemia (CLI). Methods: Five RCTs were identified after searching the Cochrane Library, MEDLINE and Embase databases from 1988 to 2013 for PTA versus PTA with stenting in patients with infrapopliteal CLI. The outcome measures were primarypatency, limb salvage, all-cause mortality and reintervention rates. Results: Six months postintervention, there was a significant decrease in lesion restenosis rates in the PTA and stent group compared with PTA alone (RR 0.6 [95\% CI 0.43-0.83]). More reintervention rates were observed in the PTA and stent group compared with PTA alone (RR 0.63 [95\% CI 0.42-0.96]). Although not statistically significant, there were fewer total limbs amputated in the PTA alone group compared with PTA and stent group (relative risk 0.58 [95\% CI 0.32-1.06]). Lastly, fewer deaths were observed in the PTA and stent group compared with PTA alone; however, this data was not statistical significant (relative risk 1.27 [95\% CI 0.65-2.50]). Conclusion: With similar limb salvage rates between PTA alone and PTA with stenting, PTA alone of infra popliteal lesions seem to provide higher patency rates and fewer re-intervention procedures in patients with CLI. Although 5 randomized clinical trials were included in this meta-analysis, we believe that larger studies with longer follow-ups are needed to properly identify the best treatment option of infrapopliteal arterial occlusive disease.

\section{7}

Diminishing returns: the relationship between repeated attempts at revascularization and major amputation: a single centre experience. M. Guirgis, ${ }^{*}$ E. Genge, ${ }^{\dagger}$ J.A. Harlock, ${ }^{*}$ D.A. Szalay. ${ }^{*}$ From the *Division of Vascular Surgery, McMaster University, and the TMcMaster University Medical School, Hamilton, Ont.

Background: Lower extremity revascularizations are performed for a number of reasons, including pain control, improved quality of life and level of function, maintenance of independence and limb salvage. These goals are met in the majority of cases but when revascularization fails a difficult decision must be made to treat conservatively (if possible), try further intervention, or proceed to amputation. Repeated failed attempts that ultimately lead to major amputation will have implications on resource utilization, may compromise the patient's overall health and wellbeing, and can significantly delay recovery and rehabilitation. This study reviews the impact and outcomes of repeated attempts at lower extremity revascularization. This is part of a larger initiative to develop a predictive model to help surgeons and patients make more informed decisions regarding these interventions. Methods: A single institution, retrospective review of consecutive patients who underwent a revascularization procedure in the operating room for peripheral arterial disease from July 2010 to June 2011, specifically looked at the number of repeat procedures/operations, limb salvage, amputation rate and mortality. Descriptive, multivariate regression and life table analyses were performed. Results: Initial results from a 1 -year pilot study found 374 limbs were treated in 338 patients. The mean age was 67 years (range $42-97 \mathrm{yr}) ; 63 \%(n=237)$ were male; $39 \%(n=146)$ were current smokers; and $43 \%(n=$ 162) were diabetics. Of the $374 \mathrm{limbs}, 81 \%(n=303)$ were treated surgically and $19 \%(n=71)$ were treated endovascularly. Indications for surgery were similarly distributed across Rutherford classes $1-3,4,5$ and 6 . Of the patients reviewed, $10 \%(n=$ 40) ultimately went on to either an above-knee amputation or below-knee amputation. Of those requiring 1 reintervention $(n=$ $71), 17 \%$ required an amputation. Of those requiring 2 reinterventions $(n=27), 52 \%$ required an amputation. Of those requiring 3 reinterventions $(n=15), 67 \%$ required an amputation. Of those requiring 4 reinterventions $(n=3), 67 \%$ required an amputation. Of those requiring 5 reinterventions $(n=2), 100 \%$ required an amputation. All-cause mortality at 3-year follow-up was $5.8 \%(n=17)$. Conclusion: Our pilot study confirms a definite relationship between number of attempts at revascularization and major amputation. We plan to further elucidate this and many other factors with our ongoing prospective study and initiative to develop a predictive model to help guide decisions about lower extremity revascularization.

\section{8}

A population-based description of the indications for lower limb amputation and associated outcomes across Canada. A. Kayssi, ${ }^{*}$ C. de Mestral, ${ }^{*}$ K. Huseynova, ${ }^{\dagger}$ G. Roche-Nagle. ${ }^{*}$ From the *Division of Vascular Surgery, 
University Health Network, University of Toronto, Toronto, Ont., and the TGuelph General Hospital, Guelph, Ont.

Background: Our objective was to describe the indications and outcomes of lower limb amputations in the Canadian population. Methods: A retrospective cohort study was carried out of all adult patients who underwent lower limb amputation in Canada between 2006 and 2009. Patients were identified from the Canadian Institute for Health Information's Discharge Abstract Database that includes all hospital admissions across Canada with the exception of Québec. Patients who underwent amputation following traumatic injury were excluded. Results: A total of 5342 patients were treated in 207 Canadian hospitals. The mean age was $67.5 \pm 13$ years and $68 \%$ were male. Amputations were most frequently indicated after diabetic complications (81\%), malignancy $(3 \%)$, osteomyelitis $(3 \%)$, or sepsis $(1 \%)$. Of the treated patients, $65 \%$ were discharged to another in-patient or longterm care facility and $26 \%$ were discharged home with or without extra support. For the total number of amputations, length of stay and discharge dispositions across providers; see Table. Conclusion: There is variability in the delivery of amputations and postoperative hospital discharges among surgical specialists across Canada. Future work is needed to investigate the reasons for this variability and develop initiatives to shorten postoperative hospitalization.

\section{9}

The pedometer and walking study (PaWS): a pilot project. N. Eisenberg, V. Kapila, D. Wooster, L. Tse, G. Roche-Nagle. From the Department of Vascular Surgery, University Health Network, Toronto, Ont.

Background: It is well known that supervised walking programs are of great benefit to patients with intermittent claudication (IC) due to peripheral arterial disease; however, such a program is not readily available in all centres due to a variety of factors. Methods: We developed a 3-month-long pilot study using 50 patients, randomized to 1 of 2 groups: pedometer or standard (walking) program. The participants were instructed to walk for a total of 30 minutes 3 times per week. Our 2 end points included change in 6-minute walking test distance and changes in ankle-brachial index (ABI) scores. We used the 6-minute walk test as a standardized outcome measure with a $25 \mathrm{~m}$ change in distance noted as clinically important. To date, 39 patients have been randomized (control $n=19$, pedometer $n=20$ ), and 31 have completed the study; within this group, 6 patients withdrew owing to disinterest or other medical conditions. No adverse events were reported. The average age of the patients is 64.4 years. Groups were generally similar in terms of gender distribution, smoking history, diabetes and hypertension. Results: Of the patient group who completed the study, those randomized to the pedometer group walked further than those not using the pedometer (average distance of $31.5 \mathrm{~m}$ and $13 \mathrm{~m}$, respectively). Participants in the pedometer group anecdotally reported less walking pain; they enjoyed the program and seeing their progress. The ABI scores did not change significantly. An unanticipated side effect has been a trend toward decreasing blood pressure over 15 points systolic, more evident in the pedometer group. Conclusion: We believe that the addition of a pedometer to conventional therapy in patients with IC is an inexpensive (under \$40) yet worthwhile addition to their therapeutic regimen based on this pilot study. A larger study is necessary to appraise its true clinical potential.

\section{0}

Management of acute limb ischemia in the pediatric population. A. Kayssi, ${ }^{*}$ F. Shaikh, ${ }^{\dagger}$ G. Roche-Nagle, ${ }^{*}$ B. Rubin, ${ }^{*}$ L.R. Brandao, ${ }^{\dagger}$ S.A. Williams. ${ }^{\dagger}$ From the *Division of Vascular Surgery, University Health Network, University of Toronto, and the tDivision of Haematology/ Oncology, Hospital for Sick Children, University of Toronto, Toronto, Ont.

Background: Acute limb ischemia (ALI) in pediatric patients is rare but may lead to limb loss and life-long complications. The aim of this study was to review the experience of a Canadian tertiary pediatric centre with the medical and operative management of ALI. Methods: The charts of in-patients diagnosed with acute upper or lower limb ischemia between 1999 and 2012 were reviewed. Patient demographics, arterial clot site and

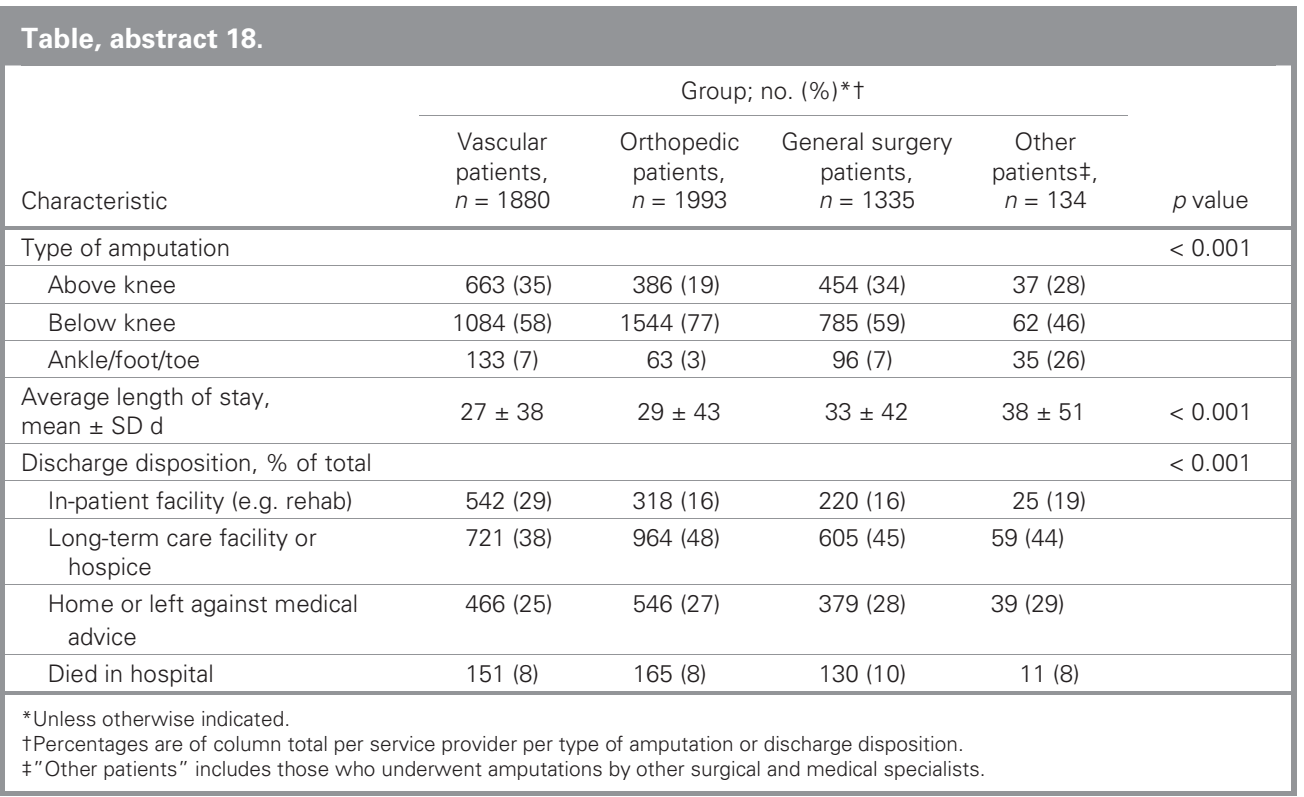


etiology, intervention, anticoagulation type and duration, and short and long-term complications were analyzed. Results: A total of 136 patients presented with signs of limb ischemia $(46 \%$ female, $34 \%$ younger than $30 \mathrm{~d}, 51 \%$ between 1 and $12 \mathrm{mo}, 15 \%$ between 1 and $18 \mathrm{yr}$ ). Of the cases, 95\% involved the lower limbs. Eighty-five percent of arterial clots were totally occlusive; $92 \%$ were due to vessel catheterization; $5 \%$ were idiopathic; and $2 \%$ were due to hereditary hypercoagulable states. Ninety-six percent were managed nonoperatively. Patients were treated with a combination of thrombolysis, unfractionated or low molecular weight heparin, aspirin and/or warfarin (duration $1 \mathrm{~d}-$ $13 \mathrm{yr}$ ). All patients were followed postdischarge at our institution or at the referring hospital (average length $3.5 \mathrm{yr}$ ). Thirteen percent had complications related to ALI or anticoagulation (e.g., limb length or thigh circumference discrepancy, or intracranial hemorrhage). A total of 25 patients died of unrelated causes (e.g., sepsis, multiorgan dysfunction or cardiac failure). Conclusion: In contrast with adults, ALI in children can generally be managed nonoperatively with anticoagulation, likely because of their greater ability to develop arterial collaterals. Long-term followup by a multidisciplinary team of pediatric and surgical specialists and allied health professionals is integral to achieving a successful outcome.

\section{1}

The role of folic acid on the hyperhomocysteinemia in the Buerger disease. A.A. Beigi, ${ }^{*}$ M.A. Hoghoughi, ${ }^{\dagger}$ A. Eshaghian, ${ }^{\neq}$A. Hassan Zade, ${ }^{\mathcal{S}}$ H. Masoudpour. ${ }^{\dagger}$ From the *Department of Vascular Surgery, tDepartment of Surgery, and ${ }^{\ddagger}$ Department of Head and Neck Surgery, Saint Al-Zahra Hospital, Isfahan University of Medical Science, and the §Isfahan University of Medical Science, Isfahan, Iran

Background: The aim of the study was to evaluate the prevalence of the hyperhomocysteinemia, the level of the anticardiolipin antibodies and the role of folic acid on the hyperhomocysteinemia and on the rate of the amputation in patients with Buerger disease (BD). Methods: In an experimental placebocontrolled double-blind study between 2004 and 2010, 30 patients with $\mathrm{BD}$ were randomly assigned to 1 of 2 groups (14 patients in drug group; 16 patients in placebo group). Exclusion criteria were those who had history of hypertension, diabetes mellitus, ischemic heart disease, cerebrovascular disease and collagen vascular diseases. In addition, they were excluded if they were given surgical treatment (e.g., sympathectomy or vascular bypass) or medical treatment (e.g., Iloprost or corticosteroid) during the study, if they used aspirin, a calcium channel blocker or vitamin B6 or B12, if they stopped smoking, or if they didn't comply with medication during the study. The drug or placebo was administered; follow-up occurred 2 and 6 months later for homocysteine, anticardiolipine antibodies and the risk of amputation. Results: At the beginning of the study, homocysteine level was higher than normal in 19 patients (63\%; normal value is $15 \mathrm{mg} / \mathrm{dL})$. There was significant decrease in homocysteine level at 6-month followup in the folic acid group $(p<0.001)$, but there was no change in the placebo group. The difference between groups was significant after the second month of the study and also in the sixth month. None of our patients had elevated anticardiolipin antibodies. Anticardiolipin antibody didn't change during 6 months of study in any groups. High level of homocysteine was not associated with more amputations during 6 months of study $(p>0.05)$. Conclusion: This study showed the hyperhomocysteinemia in $\mathrm{BD}$, and the benefit of folic acid treatment in homocysteine lowering, but folic acid didn't inhibit the risk of major and minor amputation during 6 months of follow-up. Longer follow-up may reveal the role of folic acid in these patients.

\section{SATURdaY, SePt. 14, 2013 \\ Paper session V: Venous disease \& dialysis access}

\section{2}

Outcomes after endovenous ablation for the treatment of varicose veins: a single-centre experience. A. Kayssi, M. Pope, I. Vucemilo, C. Werneck. From the Division of Vascular Surgery, Department of Surgery, Trillium Health Partners, University of Toronto, Mississauga, Ont.

Background: Our objective was to evaluate the 3-year experience with endovenous radiofrequency ablation (RFA) for the treatment of varicose veins at a single centre in Ontario. Methods: We conducted a chart review of patients who underwent RFA therapy between 2010 and 2012 at the first centre to offer this treatment modality in Canada. Patient demographics, number of limbs and vein lengths treated, radiofrequency duration and 1-month complications were noted and confirmed by duplex scan. Patients were also asked to provide feedback on the procedure using the Venous Clinical Severity Score questionnaire. Results: A total of 148 patients $(81 \%$ female, average age $54 \pm 15$ yr; 82\% CEAP2, 10\% CEAP3, 3\% CEAP4, 4\% CEAP5 and $1 \%$ CEAP6) underwent RFA between January 2010 and December 2012. In 30\% of patients, both legs were treated during the same procedure. The average length of veins treated was $42.2 \pm 21.6 \mathrm{~cm}$. The average radiofrequency duration was $218 \pm$ 113 seconds. Most patients required no pain medications or only over the counter analgesics, and only one patient required a prescription for opioid medications. The average length of time patients required before returning to work was $2.3 \pm 3.2$ days. While $92 \%$ of patients had no adverse outcomes, $4 \%$ developed skin discoloration, $2 \%$ chronic pain, $1 \%$ numbness and parasthesia, $1 \%$ developed superficial phlebitis in a tributary vein, and $1 \%$ developed heat-induced thrombosis of the common femoral vein. Occlusion of the treated veins was confirmed by duplex ultrasound scan 2 weeks after the procedure in all but 1 patient. Of the patients who provided feedback ( $45 \%$ response rate), $95 \%$ were very or extremely satisfied with the procedure. All of those patients said they would have the procedure again and would recommend it to a friend. Conclusion: Radiofrequency ablation is a safe and effective intervention for the treatment of varicose veins that is associated with a low complication rate and excellent patient satisfaction.

\section{3}

Vascular surgeon's management of venous disease in Canada: preliminary data. D.L. Wooster, ${ }^{*}$ E.F. Greco, ${ }^{*}$ S. Wong, ${ }^{*}$ E.M. Wooster. ${ }^{\dagger}$ From the ${ }^{*}$ Division of Vascular Surgery, Department of Surgery, University of Toronto and the TOntario Institute for Studies in Education/ University of Toronto, Toronto, Ont.

Background: Venous disease is the most common vascular issue encountered in the community. Vascular surgeons' (VS) involvement in venous management varies across Canada but can represent a large component of practice. Trainee involvement in the management of venous disease is often limited. With the publication of venous guidelines, interest from generalists and other 
specialists, advances in endovenous interventions, and changes in government policies, the role of VS in this issue needs attention. Our objective was to obtain an overview of VS practice patterns and attitudes related to venous disease management. Methods: A detailed electronic survey was developed similar to published surveys from other countries and sent to practising VS in Canada. The data was collated and compared with reports from elsewhere to develop a comparative management map for VS management of venous disease. Results: Eighty-two percent of respondents were in general vascular practice; $9 \%$ confined practice to a venous clinic and venous disease represented between 1 and $25 \%$ of practice in $73 \%$ of respondents. Indicators for consultation were venous ulcer (100\%), superficial vein thrombosis (SVT; $82 \%)$, and leg swelling (82\%). Increased referrals were seen over the past 5 years (55\%). Vascular surgeons treat SVT with duplex and selective treatment (64\%) or symptomatically (40\%); $82 \%$ state they follow CHEST guidelines. Vascular surgeons believe that primary care physicians (80\%) and other specialists (33\%) do not understand venous disease well. Venous-oriented continuing education (CE) represents a component of CE for $67 \%$ of VS. Specific training in venous issues was poorly represented in residency training: support stockings $(60 \%)$, sclerotherapy $(30 \%)$, EV ablation (20\%), and IVC filters (11\%). Conclusion: Preliminary data suggests that VS are involved in venous practice, they had limited training during residency and do pursue CE and include guidelines in their practice. More detailed data are require to allow a comparison to venous practice in other countries and to more fully understand training priorities.

\section{4}

Pharmacologic management of venous disease. D.L. Wooster. From the Division of Vascular Surgery, Department of Surgery, University of Toronto, Toronto, Ont.

Background: Patients with chronic venous disease, including varicose veins, can have their disorder controlled with support stockings and, in selected patients, direct interventions. Many patients present with a variety of medications, often not available in Canada, asking for advice from a vascular surgeon. Venoactive agents (VA) have been identified and are ingredients in numerous poorly standardized formulations. None are available in Canada as medically recognized pharmacologic management for venous disease; as such, knowledge of these preparations is frequently lacking. The aims of this report were to identify potential venoactive agents, describe the pharmacologic mechanism of action and the results in management of venous disease. Methods: An electronic literature search was performed to identify 1) VA ingredients in available formulations, 2) action of VA and 3) results of VA management of venous disease. Results: Over 1 million citations were returned; the majority were nonscientific. In total, $37 \mathrm{VA}$ were identified; the detailed mode of action, impact on venous disease, potential side effects and clinical outcomes were available on 5 classes of VA. These included flavonoids, rutosides, hidrosmine, escins and centella. There are 44 randomized trials showing clinical effectiveness with improvement identified in $35 \%$ to $60 \%$ for clinical appearance, symptoms of heaviness, restless legs and edema. Described side effects were limited to local sensitivity. No clear correlation of results with CEAP classification or VSS was identified. Conclusion: Certain venoactive agents have been well-studied and do show clinically efficacy. It is important to vascular specialists to be aware of them. Although the evidence is not strong, availability of consistent formulations and management recommendations could lead to improved patient care.

\section{5}

One-stage compared with 2-stage brachiobasilic fistula creation for hemodialysis access: results of a large retrospective cohort study. J. Faulds, J. Misskey, S. MacDonald, J. Chen. From the University of British Columbia, Vancouver, BC

Background: In accordance with published guidelines, at our institution brachiobasilic arteriovenous fistula creation for hemodialysis access is used when distal extremity AV fistula have failed to mature or when the patients anatomy is unsuitable for a cephalic vein fistula. Brachiobasilic transposition can be performed in a single operation, or can be staged and completed in 2 operations. The purported benefits of a single stage approach include a shorter duration of catheter-based dialysis, and the convenience of a single operation. Recent reports have suggested that a staged approach results in reduced nonmaturation rates and improved fistula patency. Although the trend at our institution is toward a 2 -staged approach, there remains clinical equipoise as to the best method of brachiobasilic AV fistula creation. The primary goal of this study is to determine if a 2-staged approach leads to reduced primary failure rates when compared with single stage brachiobasilic fistula creation. Secondary goals are to determine differences in primary patency, secondary patency and overall complication rates between cohorts. Methods: A review of all patients requiring brachiobasilic arteriovenous fistulae for hemodialysis access at a single University between 2005 and 2012. The study was designed as a retrospective cohort study with patients divided into single stage or planned 2-stage cohorts. All patients having attempted arteriovenous fistula creation, even those that never require hemodialysis, are captured within our prospectively maintained renal access database. The database was reviewed by 2 reviewers and captured all patients who had a brachiobasilic fistula at 2 large academic hospitals. Results: A total of 169 patients underwent attempted brachiobasilic arteriovenous fistula creation during the years of the study. Sixty-nine patients had the fistula created at a single operation and formed the single stage cohort. One hundred patients had the fistula created using a preplanned 2-stage approach and formed the 2-stage cohort. There was no difference in the primary end point of nonmaturation, with $23.2 \%$ of single stage patients and $27.0 \%$ of 2 stage patients failing to achieve a functional fistula $(p=0.567)$. At 1 year, $64.1 \%$ of patients in the single stage cohort were dialyzing through their fistula compared with $59.3 \%$ of 2 -stage patients $(p=0.532)$. At 2 years, $53.1 \%$ of single stage patients were functional, compared with $44.1 \%$ of 2 -stage patients $(p=0.07)$. Conclusion: Despite a recent trend toward using a 2-stage approach for brachiobasilic fistula creation, this large, prospective cohort study was unable to detect any difference in maturation and fistula patency between the single stage and 2-stage approach.

\section{SATURDAY, SEPT. 14, 2013 \\ PAPER Session VI: General VAscular topics}

\section{6}

Comparison of cerebral oximetry and transcranial doppler in decision to shunt for carotid endarterectomy. J. Dooner, S. Lee, C. Dooner. From Victoria, BC

Background: Conducting carotid endarterectomy under general anesthetic compromises cerebral function monitoring compared 
with operating on the awake patient. Surrogate systems include EEG, transcranial Doppler (TCD) and stump pressure (SP) monitoring. Cerebral oximetry is another modality that has been used in other settings but has not had widespread use in carotid surgery. Our objective was to evaluate the use of cerebral oximetry $(\mathrm{COX})$ against more established technology, TCD and SP and determine if it correlates adequately to act as a stand alone monitor and decision tool for selective carotid shunting. Methods: A consecutive series of patients presenting for carotid endarterectomy were analyzed prospectively. Fifty patients were studied and 45 complete data sets were analyzed (see Table). Five patients were excluded due to inability to find a temporal window allowing TCD monitoring. Decision to shunt was based on transcranial Doppler response based on a long standing protocol in our institution. Reduction of mean TCD velocity of $50 \%$ or greater following clamping would dictate the use of an indwelling shunt. Results: There were 32 male patients and 13 female patients. Average age was 71.3 years. Thirty 5 patients were symptomatic 10 were asymptomatic. Conclusion: Cerebral oximetry correlated well with the SP (mean) and showed a significant difference in the shunted and nonshunted patients $(p<0.05)$ that would allow it to be used as a tool in a selective shunting decision algorithm. More information is needed to explore the sensitivity of this technique.

\begin{tabular}{|c|c|}
\hline Characteristic & Mean $\pm S D^{*}$ \\
\hline \multicolumn{2}{|l|}{ Sex, no. } \\
\hline Male & 32 \\
\hline Female & 13 \\
\hline Age, yr & 71.3 \\
\hline Symptomatic, no. & 35 of 45 \\
\hline Shunt used, no. & 20 of 45 \\
\hline Postoperative stroke, no. & 0 \\
\hline Postoperative death, no. & 0 \\
\hline \multicolumn{2}{|l|}{ Cerebral oximetry } \\
\hline Baseline & $53.8 \pm 20.9$ \\
\hline Post-clamp & $65.5 \pm 11.93$ \\
\hline$\%$ change post-clamp & $0.99 \pm 0.16$ \\
\hline $\begin{array}{l}\text { SD }=\text { standard deviation. } \\
{ }^{*} \text { Unless otherwise indicated. }\end{array}$ & \\
\hline
\end{tabular}

27

Relationship between popliteal artery aneurysm size and symptomatic status. S.V.B. Patel, S.V. Patel, L. Dubois, A.H. Power, J.R. Harris, G. DeRose, T.L. Forbes. From the Division of Vascular Surgery, Western University, London, Ont.

Background: Our objective was to compare the presence of symptoms in patients undergoing popliteal aneurysm repair in a tertiary care centre for small $(<4 \mathrm{~cm})$ and large $(>4 \mathrm{~cm})$ aneurysms. Methods: Using a prospectively collected vascular surgery database at a tertiary referral centre, a retrospective cohort study was performed. All patients who had surgical repair of popliteal aneurysm were included from 2003 to 2012. The presence of symptoms was compared between large aneurysms $(>4 \mathrm{~cm})$ and small aneurysms $(<4 \mathrm{~cm})$. The size of aneurysm, presence of mural thrombosis and distal run-off were also compared between symptomatic and asymptomatic patients. Short and long-term reintervention and amputation rates were also compared between these groups. Results: A total of 68 patients undergoing popliteal aneurysm repair were enrolled with 43 having information about symptoms. There were 20 patients who were asymptomatic and 23 patients who reported symptoms of claudication, compression or ischemia. There was no difference in the proportion of symptoms in small compared with large aneurysms $(52.1 \%$ small, $55.0 \%$ large, $p=$ $0.85)$. There was no difference in the size of aneurysms between symptomatic and asymptomatic groups $(p=0.59)$. There was an increased proportion of symptomatic patients with mural thrombosis $(p=0.045)$ or a history of smoking $(p=0.052)$. Amputation rates at 2 years were considerably higher in the symptomatic patients (asymptomatic 0\%, symptomatic 20\%, $p=$ $0.03)$. Conclusion: The belief that popliteal aneurysm size and progression to symptoms has been debated. Despite this belief we have shown that the size of aneurysm is not associated with symptoms even when larger than $4 \mathrm{~cm}$. Furthermore, our data supports the mounting evidence that aneurysm characteristics and patient comorbidities contribute to the likelihood of developing symptoms in popliteal aneurysms.

\section{8}

Visceral artery aneurysm/pseudoaneurysm management: comparison of endovascular and surgical treatments. S. Galante, ${ }^{*}$ G.D. Oreopoulos. ${ }^{*}+$ From the ${ }^{*}$ Division of Vascular Interventional Radiology, Joint Department of Medical Imaging, University Health Network, University of Toronto, and the tDivision of Vascular Surgery, Department of Surgery, University Health Network, University of Toronto, Toronto, Ont.

Background: Visceral artery aneurysms and pseudoaneurysms (VAA) are rare clinical entities that can be treated by surgical or endovascular means. The purpose of the study was to evaluate the clinical features, imaging, treatment, clinical outcomes, and to determine the dominant mode of treatment for VAA at our institution. Methods: From January 2009 to January 2012, 30 aneurysms were treated in 28 patients (12 male, 16 female, mean age $55 \mathrm{yr})$ at our institution. Endovascular treatment was used for 26 patients; arteries involved were splenic (13 cases), superior mesenteric (2), hepatic (3), gastroduodenal (2), renal (5), and gastroepiploic (1). Surgical resection was used in 2 cases: 1 involving the superior mesenteric artery and 1 involving the confluence of the gastroduodenal and common hepatic arteries. Indications for treatment included incidental (size $>2 \mathrm{~cm}$ diameter; 12 cases); bleeding/rupture (7); postsurgical/posttraumatic (4); familial/ vasculopathy (4); and hypersplenism secondary to portal hypertension (1). Endovascular treatments included coils alone (11), coils and cyanoacrylate glue (7), coils and absorbable gelatin sponge (3), Amplatzer plug, cyanoacrylate glue, and coils (1), stent grafting (2), and direct sac puncture with glue injection (3). Follow-up based on clinic visits or diagnostic imaging was available in 24 of the 28 patients with a mean follow-up time of 11 months. Results: Twenty-three of the 24 patients with followup had successful treatment of their aneurysms. Of those, $16 \mathrm{had}$ no complications, 5 had asymptomatic areas of target organ infarct noted on imaging; 1 thrombosed aneurysm became infected requiring drain insertion; 1 developed pancreatitis with a pseudocyst requiring surgical drainage. The 24th had incomplete treatment of their renal artery aneurysm owing to nontarget embolization of glue during the procedure requiring placement of an aortic stent graft. The 2 surgically treated patients had successful treatment of their aneurysms, 1 patient (hepatic artery/gastroduodenal aneurysm) had asymptomatic areas of hepatic infarct on imaging. Conclusion: Endovascular treatment of VAA is safe, effective, and now the dominant method of 
treatment at our institution. However, surgical management still has a role in certain cases.

\section{9}

Prevalence of carotid body tumour in a vascular laboratory in Bogota, Colombia. A. Munoz. From the Clínica Vascular de Bogota, Universidad Nacional de Colombia, Bogota, Colombia

Background: My objective was to identify carotid body tumour (CBT) prevalence in the vascular laboratory of Bogota Vascular Clinic. In Bogota, a city located over $2000 \mathrm{~m}$ over sea level, surgery for this condition is more frequent than carotid endarterectomy. Methods: A retrospective single site study was conducted. The study population consisted of patients referred for carotid duplex scanning examinations from September 2007 to September 2012. Patient demographics, symptoms and duplex results were analyzed to estimate the prevalence and size of carotid body tumours, and assist patients with incidental small tumours. Results: Over the past 6 years, we performed 3.589 carotid duplex scanning examinations in our vascular laboratory. A total of 128 patients with 139 tumours were diagnosed with a new CBT, with a prevalence of $3.61 \%$. The patients were referred for palpable mass in $88(69 \%)$ cases and for suspected carotid artery disease in $32(31 \%)$ cases. Of these patients, 15 $(11.7 \%)$ were men and $113(88.3 \%)$ women. The mean age was 59.7 years (range 26-91). Sixty (46.8\%) tumours were located on the right side, 57 (44.5\%) on the left side and 11 (8.7\%) bilateral. The mean tumour size was $2.47 \mathrm{~cm}$ (range 0.64-5.19). Thirtynine $(28.4 \%)$ tumours were less than $2 \mathrm{~cm}$; 95 were $(68 \%)$ between 2.0 and $5.0 \mathrm{~cm}$; and $5(3.6 \%)$ larger than $5 \mathrm{~cm}$. Twentyseven patients with incidental small tumours have not received surgical treatment and are currently being followed with yearly duplex scan in our vascular laboratory. Conclusion: Duplex scanning is a frequent examination to diagnose carotid artery disease. The prevalence of incidental CBT in vascular laboratories of high altitude cities is a trend. Diagnosis of small nonpalpable tumours is more frequent. As surgical resection has been recommended for CBT independent of size, and even small tumour resection is associated with complications, further research of high altitude carotid body tumours is necessary to better determine the surgical indications.

\section{FRIDAY, SEPT. 13, 2013 CSVS POSTER SESSION}

\section{0}

Accuracy of centerline of flow measurements for sizing endovascular grafts and centerline angles, which are predictive for inadequate sizing. K. Lee, T.L. Forbes, J.R. Harris, G. DeRose, A.H. Power. From the Division of Vascular Surgery, Western University, London, Ont.

Background: Our objective was to determine the accuracy of centreline of flow measurements for sizing endovascular grafts. Centerline angles, as a measure of aortoiliac tortuosity, were explored for their predictive value of inadequate preoperative sizing. Methods: Fifty consecutive patients treated by endovascular aneurysm repair in 2012 were retrospectively analyzed using TeraRecon centreline software. Results: Fifty patients underwent aorto-uni-iliac and aorto-bi-iliac repairs (9 and 41 patients, re- spectively) using the following stent grafts: 36 Medtronic Endurant, 12 Cook Zenith, and 2 Vascutek Anaconda. A total of 91 pre- and postoperative lengths from lowest renal artery to internal iliac artery were obtained. As well, preoperative infrarenal, aortoiliac, and common iliac centreline angles were determined. Overall, there was no significant difference between the pre- and postoperative lengths $(p=0.37$, mean difference $3 \mathrm{~mm})$. However, 12 postoperative lengths (13\%) were at least $10 \mathrm{~mm}$ (or $5 \%$ ) shorter than their respective preoperative lengths. All of these had aortoiliac $(n=5)$ or common iliac $(n=8)$ centreline angles greater than $90^{\circ}$. Only 1 centreline had a common iliac angle greater than $90^{\circ}$ and no significant change in pre- and postoperative length measurements. There were no infrarenal centreline angles greater than $90^{\circ}$. Conclusion: Length calculations based on centreline measurements provided accurate lengths for sizing endovascular grafts in the majority of cases. However, preoperative aorto-iliac or common iliac centreline angles greater than $90^{\circ}$ were associated with shorter postoperative lengths, suggesting that placement of shorter grafts should be anticipated.

\section{1}

Application of Enhanced Recovery After Surgery (ERAS) in Canadian vascular surgery. M.C. Rockley, H. Cox, J.P. Bayne. From the University of Alberta, Edmonton, Alta.

Background: Our objective was to understand the current state of perioperative care in Canadian vascular surgery, to investigate the barriers to integrating Enhanced Recovery After Surgery (ERAS) perioperative concepts into Canadian vascular surgery, and to assist surgeons in optimizing their perioperative management. Methods: An online survey concerning the aspects of perioperative management addressed by ERAS was sent to all CSVS members. Each participant will receive a comparison of his/her centre with both the national standard and published evidence. Results: Early results have yielded a 16\% response rate; we are awaiting further results before final analysis. Current respondents reported postoperative management which reflects ERAS in the following areas: avoidance of bowel preparation $(95 \%)$, early mobilization (95\%), routine epidural analgesia $(95 \%)$, routine avoidance of abdominal drainage $(86 \%)$, and routine postoperative antiemetics (52\%). Respondents reported postoperative management which does not reflect ERAS in the following areas: preoperative fasting timeline (5\%), postoperative gum chewing $(11 \%)$, early removal of Foley catheters despite epidural analgesia (29\%) and avoidance of postoperative nasogastric decompression (33\%). There are multiple reasons why practice may not resemble current evidence. Of the respondents who use postoperative nasogastric decompression, $50 \%$ are not aware of contrary evidence, while $43 \%$ do not find the contrary evidence to be adequate. Thirty-nine percent do not find evidence supporting postoperative gum chewing to be adequate. As well, $46 \%$ of respondents reported not removing Foley catheters during epidural analgesia despite believing that it is safe, citing external barriers preventing change in their practice. Conclusion: Enhanced Recovery After Surgery includes a collection of evidence-based perioperative guidelines. Although developed for general surgery, perioperative similarities allow guidelines to be applied effectively in vascular surgery. Several concepts of ERAS are prevalent in Canadian vascular surgery, while others have not been incorporated. Where practice differs from ERAS, respondents cite both a lack of awareness of the literature and assertion that current literature is inadequate. To promote awareness, we will provide literature summaries to respondents including vascular specific trials, and further disseminate the literature nationally. Where 
evidence is inadequate, we plan to perform randomized controlled trials to clarify discrepancies.

32

Early versus late enteral nutrition and associated clinical outcomes in critically ill patients after thoracic and abdominal aortic surgery. A. Ho, A. Smith, R. Stallard, K. Vandenbussche, G. Papia. From the Department of Critical Care Medicine, Division of Vascular Surgery, Sunnybrook Health Sciences Centre, Toronto, Ont.

Background: Our objective was to examine the timing of initiating enteral nutrition (EN) in critically ill patients after aortic surgery in our cardiovascular intensive care unit (CVICU) in comparison to current Canadian practice guidelines, and to evaluate any associated morbidity and mortality. Methods: Between January 2004 and December 2011, all patients after open and/or endovascular repair for aneurysmal or occlusive aortic disease were screened for use of $\mathrm{EN}$ in the CVICU. A retrospective chart review was completed on eligible patients. All critical care intensivists and vascular surgeons were surveyed on their practices regarding initiating EN in this patient population. Results: A total of 1011 patients were screened and 34 (5\%) received postoperative EN in CVICU. Of those who received $\mathrm{EN}$, only $15 \%$ received early EN (within $24-48 \mathrm{~h}$ of admission). The most common reason for late initiation of $\mathrm{EN}$ was reinitiation of mechanical ventilation. There were no significant differences in morbidity and mortality between early and late EN groups. On subgroup analysis, patients on inotropes who received early EN had significantly lower mortality and fewer infections. Seventy-three percent of critical care intensivists surveyed start early EN, while $67 \%$ of vascular surgeons wait for signs of bowel function. Despite differences in practice, $86 \%$ of physicians feel that current practice guidelines for early EN are applicable to these patients. Conclusion: Most postoperative aortic surgery patients in our CVICU did not require EN. However, for those that did, practice guidelines were not achieved. Achieving early EN in sicker patients was associated with better outcomes. Practices between intensivists and surgeons regarding $\mathrm{EN}$ varied.

\section{3}

Endovascular aortic aneurysm repair surgical waste audit. N. Bowers, ${ }^{*}$ L. Tse, ${ }^{\dagger}$ N. Eisenberg, ${ }^{\dagger}$ G. Roche-Nagle. ${ }^{\ddagger}$ From the *University of Toronto, tDepartment of Vascular Surgery, ¥Toronto General Hospital, Division of Vascular Surgery, University Health Network, Toronto, Ont.

Background: The University Health Network adopted an environmental management system in 2000 to identify opportunities to "green" the hospital. Operating rooms (ORs) are estimated to generate up to one-third of hospital waste. To investigate waste production associated with EVARs, we performed a surgical waste audit to gauge the environmental impact of this procedure and generate strategies to improve waste management. Methods: We conducted a waste audit of 5 EVARs performed by the vascular department in February 2013. Waste was categorized into 6 streams: regular solid waste, recyclable plastics, recyclable paper, biohazard waste, laundered linens and sharps. Volume and weight of each stream was quantified. We used Canadian hospital discharge abstract data (2008-2009) to estimate annual weight and vol- ume totals of waste from all EVARs performed in Canada. Results: The average surgical waste (excluding laundered linens) per EVAR was $20.4 \mathrm{~kg}$, of which $13.1 \mathrm{~kg}(64.2 \%)$ was normal solid waste, $2.62 \mathrm{~kg}(12.8 \%)$ was biohazard waste, $2.44 \mathrm{~kg}(12 \%)$ was device boxes, $2.06 \mathrm{~kg}(10.1 \%)$ was recyclables and $0.18 \mathrm{~kg}(0.9 \%)$ was sharps. The average volume of waste per EVAR was $0.77 \mathrm{~m}^{3}$. Device packaging contributed considerably to total waste. We estimated that landfill waste from all 3756 EVARs performed in Canada in from 2004 to 2009 was $76662 \mathrm{~kg}$ in weight and $2895.5 \mathrm{~m}^{3}$ in volume. Conclusion: Endovascular aortic aneurysm repairs produce considerable amounts of surgical waste. Environmentally friendly surgical products and waste management policies may allow ORs to diminish the negative effects of waste production without compromising patient care.

\section{4}

Have guidelines altered vascular ultrasound practice? D.L. Wooster. From the Division of Vascular Surgery, Department of Surgery, University of Toronto, Toronto, Ont.

Background: Numerous clinical practice guidelines (CPGs) and testing and interpretation standards that impact on vascular ultrasound (VU) have been recently published. Accrediting agencies (e.g., College of Physicians and Surgeons of Ontario) and payers (e.g., Ministry of Health and LongTerm Care) have called for increased applications of guidelines. Experience in practice, however, suggests that there is limited uptake of such guidelines by vascular surgeons and imaging specialists involved with VU. The aims of this study were to 1) identify CPGs and standards relevant to VU practice, 2) determine the level of their incorporation into protocol manuals and 3) correlate the impact of this on interpretation reports sent to referring physicians. Methods: An electronic literature review was performed to identify CPG and standards; the findings were correlated with appropriate testing and description of test protocols and reporting criteria. Protocol manuals were obtained and parsed for evidence of incorporation of guidelines. Interpretation reports were subjected to a structured audit tool designed to identify guideline-driven reporting. Results: Ten protocol manuals were reviewed; $70 \%$ indicated guidelines were used in the manual (20\% consistently cited and $50 \%$ limited reference). Guidelines were cited more frequently in carotid (70\%) and arterial (40\%) testing than in venous testing (20\%). In 100 interpretation reports, overall reporting standards were followed in $68 \%$ of cases. Testing standards were followed in $68 \%$ but surveillance recommendations in only $30 \%$. Evidence of guideline-driven reporting varied with individual test modalities (carotid $78 \%$, arterial $42 \%$, venous $22 \%$ ). Conclusion: Incorporation of published guidelines relevant to vascular ultrasound testing into protocol manuals remains incomplete. Interpretation reports do not reflect guidelinedriven practice, particularly in venous and arterial studies. Although a larger scale study is required to identify the impact on patient care, emphasis on guideline implementation remains an important priority in quality vascular care.

\section{5}

Initial experiences with a novel international vascular surgery trainee exchange program. J.A. Harlock, R. Gowing, T. Rapanos, J.G. Tittley, D.A. Szalay. From McMaster University, Hamilton, Ont. 
Background: Direct entry into vascular surgery training programs has proven to be a popular and successful initiative in the United States, and has recently been instituted in Canada. Residency training programs on both sides of the border now have the mandate of ensuring their trainees are fully prepared for contemporary and independent practice in 5 years. In the United States, the graduating trainee average number of open vascular cases (GTAN) continues to decline. Moreover, integrated vascular trainees have less exposure to open abdominal surgery compared with traditional Independent trainees. Currently, Canadian vascular surgery trainees have less emphasis on endovascular techniques than their American counterparts. Here we present a novel Accreditation Council for Graduate Medical Education (ACGME)-approved US-Canada exchange program aimed to improve both open vascular and endovascular training for both cohorts. Methods: From 2010 to 2012, 2 integrated vascular residents from Stanford University Medical Center (Palo Alto, Calif.) and 2 vascular fellows from McMaster University (Hamilton, Ont.) participated in a 3-month clinical exchange rotation. Procedure logs were compared from exchange period and with the GTAN for 2011. Results: The USV performed an average of 141 procedures during the exchange period, 91 (65\%) being primary open reconstructions. This included 10 open abdominal aortic aneurysm (AAA) repairs, (v. 12 GTAN), 2 open ruptured AAA repairs (v. 3.4 GTAN), 5 aortobifemoral bypass (v. 9 GTAN), and 11 femoral-popliteal bypass (v. 19 GTAN). Canadian vascular surgery trainees performed an average of 69 procedures, with $51(74 \%)$ primary endovascular interventions. Conclusion: This ACGME/American Board of Surgeryapproved international surgical exchange program provides complementary training opportunities to both institutions not available outside this framework. Focused as a concentrated elective rotation, similar exchanges may serve as a model for supplementing training in both countries.

\section{6}

Patterns of prescribing vascular risk-modifiers among Australian, Canadian, New Zealander and South African vascular surgeons. A. Kayssi, ${ }^{*}$ C.B. Beiles, ${ }^{+}$ G. Roche-Nagle. ${ }^{*}$ From the *Division of Vascular Surgery, University Health Network, University of Toronto, Toronto, Ont., and tWestern Hospital, University of Melbourne, Melbourne, Australia

Background: Several guidelines have been developed to help clinicians optimize vascular risk factors such as diabetes, dyslipidemia, hypertension, and smoking. The goal of this study was to assess vascular surgeons' views on prescribing vascular risk factor medications (RFs) in 4 countries. Methods: An online survey was administered to vascular surgeons in Australia (AU), Canada (CA), New Zealand (NZ) and South Africa (SA) to investigate their attitudes toward prescribing RFs and identify barriers to improving patient compliance. Results: Ninety-one surgeons completed the survey (32 AU, 33 CA, 7 NZ, 19 SA) with an average career span of 17 years. Most CA surgeons had an academic practice $(51.5 \%$ ), while less than $50 \%$ of other respondents had a private or mixed academic/private practice. A total of $54.9 \%$ of respondents believed that most of their patients were on appropriate RFs. A total of $69.2 \%$ routinely prescribed antiplatelets (89\%) and statins (73.6\%), but only $22 \%$ regularly monitored side effects. While $57.9 \%$ of SA surgeons believed that surgeons should be primarily responsible for prescribing RFs, most other respondents disagreed. Most respondents believed that vascular surgeons relied on their medical colleagues to prescribe RFs (67\%) and did not want to monitor side effects
(68.1\%). A total of $82.4 \%$ believed it was possible to improve patient compliance and identified barriers such as lack of patient understanding of medication benefits (74.7\%), polypharmacy $(52.7 \%)$, and side effects $(42.9 \%)$. Conclusion: Our findings suggest that most vascular surgeons in the countries surveyed prescribe some RFs but expect primary health care providers and medical specialists to prescribe other RFs and monitor patient compliance and side effects. Compliance can be improved through better patient education on medication benefits and closer collaboration with other physicians to monitor and treat medication side effects.

\section{7}

Perceptions of current and previous vascular surgery residents regarding future job opportunities. J.A. Cooper, ${ }^{*}$ L. Dubois, ${ }^{*}$ A.H. Power, ${ }^{*}$ J.R. Harris, ${ }^{*}$ G. DeRose, ${ }^{*}$ K.S. MacKenzie, ${ }^{\dagger}$ T.L. Forbes. ${ }^{*}$ From the * Division of Vascular Surgery, Western University, London, Ont. and tMcGill University, Montréal, Que.

Background: Our objective was to determine the current employment environment for recent vascular surgery graduates and to identify factors that facilitate their job prospects. The perceptions of current trainees in regards to potential employment opportunities are also explored. Methods: A descriptive, cross-sectional web-based survey of Canadian residents currently enrolled in vascular surgery training programs, and of recent graduates (2011 and 2012). Results: Preliminary results include responses from 14 current residents and 5 recent graduates. Sixty percent of recent graduates felt that employment opportunities was a very important factor in the decision to pursue a career in vascular surgery, compared with only $14 \%$ of current trainees. All of the recent graduates attained employment, with $80 \%$ doing so before completing their training. On average, each graduate received 2.6 job offers and $100 \%$ of graduates obtained the position they desired. In contrast, $57 \%$ of current trainees foresee it being difficult to find employment in vascular surgery, and predict that they might have to extend training due to lack of positions. Oversaturation of the job market and overproduction of trainees were the most important concerns of both graduates and trainees with regards to attaining employment. Conclusion: There is a discrepancy between the favourable employment climate experienced by recent Canadian vascular surgery graduates and the grave outlook of current trainees. Recent graduates have enjoyed multiple job offers and $100 \%$ employability, while current trainees fear the potential effects of too many trainees and an oversaturated job market. For this favourable employment climate to persist, Canadian vascular surgery must be progressive in balancing the employment opportunities with the number of graduates. Number and timing of graduate job offers is a possible future metric of the optimum number of residents.

\section{8}

Retrospective and prospective review of abdominal EVAR cases at Sunnybrook Health Sciences Centre, type II endoleaks: a decade of experience and lessons learned. K. Maggisano. From Sunnybrook Health Sciences Centre, Toronto, Ont.

Background: The purpose of this study was to review our experience with EVAR in order to delineate the incidence of type II endoleaks and their associated risk factors. Methods: A retrospective review of 335 abdominal procedures requiring endovascular grafts was performed. Descriptive statistics were calculated to compare early and late cohorts. Medians were compared using the 
Mann-Whitney $U$ test as were categorical variables using the $\chi^{2}$ or Fisher exact test, as appropriate. Results: In comparing early (2004-2008) to late cohorts (2009-2012), median patient age, sex ratios, and the incidences of diabetes mellitus, hypertension, dyslipidemia and anticoagulant use were similar. Smoking was more prevalent in the late group. Maximal transverse sac size was similar in both, as was the landing zone size; although, the early group had larger neck sizes. The majority of type II endoleaks were diagnosed intraoperatively (53 of 88, 60\%), 25\% (22 of 88) within the first year of follow-up, $15 \%$ after 1 year (13 of 88). The type II endoleak rate was similar in both the early and late cohorts $(23.5 \%$ v. $27.0 \%$; OR $1.19,95 \%$ CI $0.64-2.31$; $p=0.66)$. Univariable analysis revealed that the graft from one manufacturer was at lower odds (OR $0.55,95 \%$ CI $0.31-0.93 ; p=0.03$ ) of type II endoleak. Multivariable analysis demonstrated that no variables achieved statistical significance for predicting type II endoleak. Conclusion: Type II endoleaks are usually found in the operative period and during the first year of follow up. Beyond this point the incidence rate (13 of 335) of them was relatively low (3.9\%). The overall type II endoleak rate in this study was $26.2 \%$ (88 of 335 ). Their incidence in this patient group did not decrease over time, despite evolved operator technique and experience.

\section{9}

Same-day discharge following percautanoues endovascular aneurysm repair (PEVAR): a single centre experience and related outcomes. I. Vucemilo, C. Werneck,
W. Johnson, M. Pope. From the Trillium Health Partners, Mississauga, Ont., and the Division of Vascular Surgery, Department of Surgery, University of Toronto, Toronto, Ont.

Background: There is an ever increasing trend toward minimally invasive surgical procedures. Total percutaneous access for EVAR has been reported to be safe and effective with acceptable success rates in the literature. Here we present a single centre experience with same-day discharge following percautanoues EVAR (PEVAR) for abdominal aortic aneurysm (AAA) repair using the Perclose device. Methods: A retrospective cohort study was completed, comprising patients who underwent PEVAR at a single centre since March 2010 and discharged home on the same day of the surgery. Patient characteristics, operative time, local and systemic complications, and rate of endoleak were recorded and analyzed prospectively. Results: In total, 10 patients who underwent PEVAR since March 2010 were discharged home on the same day. The majority of patients were male (9 of 10), with an average age of 72 years and average size of AAA of $6.1 \mathrm{~cm}$ (range 5.0-8.4). The mean surgical time was 91 minutes. There was note of groin bleeding requiring open exploration in the OR. There were no wound infections, pseudoaneurysms or delayed bleeding after PEVAR with Perclose device. There was only 1 type II endoleak noted during postoperative follow-up, and it was managed conservatively. Conclusion: Percutaneous EVAR may decrease surgical time and hospital stay compared with surgical cut-down without an increase in local complications. The extra cost of a Perclose device may be justified. Same-day discharge may be a safe and reasonable option in the right patient population. 